\title{
The influence of combined VVT systems on polluting emissions and dynamic performance in SIE
}

\author{
Daniel LITA ${ }^{1}$, Florian IVAN ${ }^{2}$ \\ ${ }^{1}$ Renault Technologie Roumanie; ${ }^{2}$ University of Pitesti, Romania \\ *Corresponding author e-mail: florian.ivan@upit.ro
}

Article history

Received 11.05.2020

Accepted 15.07.2020

DOI https://doi.org/10.26825/bup.ar.2020.013

\begin{abstract}
One of the modern methods of reducing pollutant emissions and increasing dynamic performance is the implementation of distribution phase variation systems for both the intake valve and the exhaust valve, called combined VVT, applied over a wide range of engine operation regimes. The paper aims to highlight how such a solution leads to improved dynamic performance and reduced chemical pollution. Achieving this goal involved experimental investigations into a small class cars SIE. The variants of combined VVT (intake \& exhaust), research methodology and an analysis of experimental results on a wide range of functional regimes are presented. It is concluded that such a solution can lead by implementation on engines to remarkable results both in terms of NOx reduction and in terms of dynamic performance (torque and power).
\end{abstract}

Keywords: VVT intake \& exhaust, nitrogen oxides, dynamic performance.

\section{The paper's objectives}

The combined VVT (intake \& exhaust) is the realization of distribution phases optimized for both intake and exhaust, the optimization being performed depending on the operating mode of the engine, depending on load and speed. There are 4 cases of variation followed during the research in the field, namely:

a. Maximum overlap - the variant that implies a maximum overlap duration of the inlet and outlet valve opening (case shown in figure 1, where in red is shown the lift height of the exhaust valve, and in blue the lift height of the intake depending on the angle of rotation of the crankshaft);

b. Minimum overlap - the variant that involves a minimum duration (to 0 ) of overlapping the opening of the intake and exhaust valves, as shown in figure 2;

c. Maximum delay - which implies a variation of the phases shown in figure 3;

d. Maximum advance - which implies a variation of the phases shown in figure 4. 


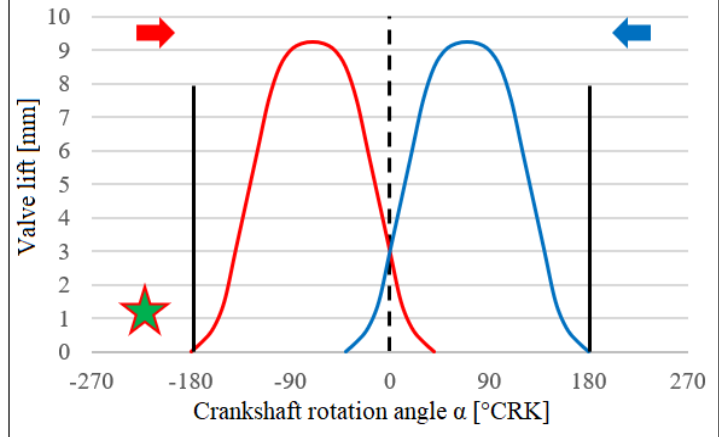

Figure 1. The case of maximum overlap

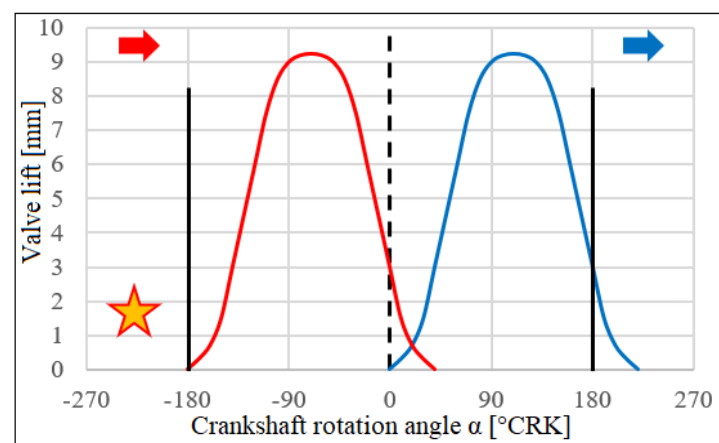

Figure 3. The case of the maximum delay

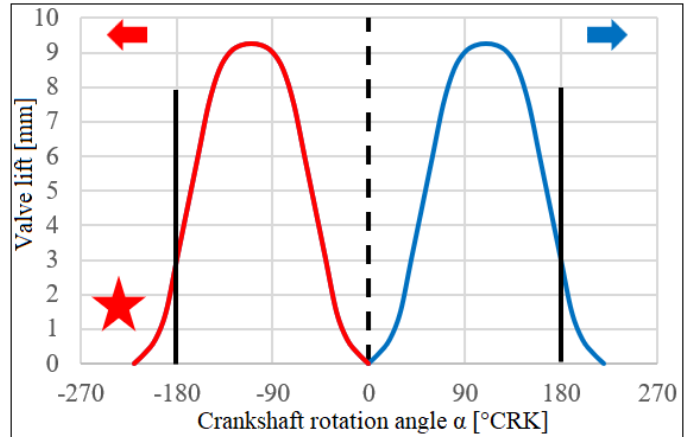

Figure 2. The case of minimal overlap

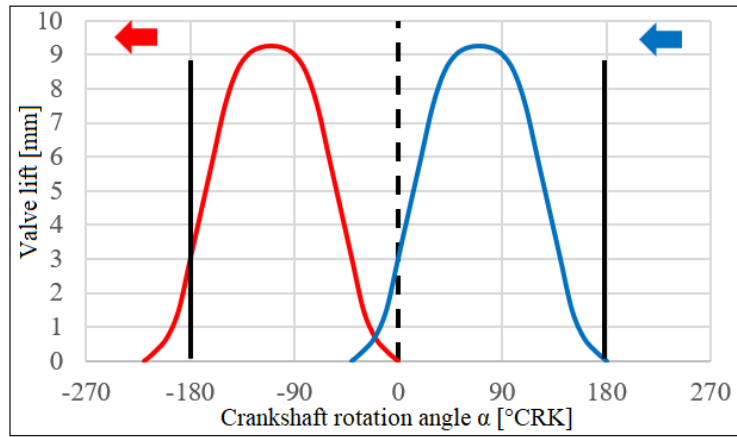

Figure 4. The case of the maximum advance

\section{Phenomenology and research methodology}

In the first case -maximum overlap- shown in Figure 1, the aim is to reduce NOx emissions by creating the so-called flue gas recirculation phenomenon called internal EGR. However, it should be noted that such a methodology must be applied with caution as a maximum overlap can lead to the occurrence of the reverse flow phenomenon, called "scavaging". This phenomenon involves the flow of fresh cargo from the intake valve gate to the exhaust valve gate. As a result, the chemical composition of the flue gases may be unduly altered, leading to incorrect information of the oxygen sensor on the actual oxygen content resulting from the combustion process. This phenomenon is often encountered in the area of low speeds and high loads, as the long running time of the engine cycle combined with the high pressure lead to a high gas exchange between the intake and exhaust valves.

On the other hand, the option of maximum overlap involves two major advantages:

- the first advantage refers to the reduction of the delay of closing the exhaust valve so that at low speeds the flow of fresh load in the intake manifold is avoided, at the beginning of the compression stroke, thus avoiding the degradation of the engine filling efficiency;

- $\quad$ the second advantage is to reduce the advance when opening the exhaust valve, in the area of low and medium loads and speeds, such a solution is used to make the most of the energy generated by the gases on the expansion stroke, respectively reducing pumping losses with favorable repercussions related to increasing dynamic and economic performance.

The second situation, the case of minimum overlap, shown in Figure 2, has three major advantages:

- the increased advance at the opening of the exhaust valve leads to the decrease of the pumping losses due to the increase of the free exhaust phase of the flue gases;

- a minimum overlap leads to a reduced amount of residual flue gas re-sucked into the cylinder, which causes both an improved filling of the cylinder and an increased homogenization of the mixture;

- an increased delay in closing the intake valve is beneficial for inertial filling of the cylinder, especially at high speeds, a condition imposed by the manufacturer primarily to achieve high peak dynamic performance. 
The third possibility of optimizing the combined VVT, maximum delay, shown in Figure 3 leads to two important advantages at low and medium speeds and loads, namely:

- the first advantage consists in the more efficient fructification of the gas energy on the expansion stroke, decreasing the duration of the free evacuation phase, an advantage borrowed from the first case;

- the second advantage is the reduction of NOx emissions through the phenomenon of internal EGR. The two advantages offered partially or totally exclude the disadvantages registered in the first variant.

The fourth situation, maximum advance, shown in Figure 4, does not present significant advantages in terms of dynamic engine performance and NOx reduction, which is why it is not applicable.

In order to highlight the influence of VVT intake and exhaust on the engine stand, a number of 84 stabilized functional regimes were investigated (respectively 12 speed regimes and 7 load regimes), summarized in tables 1 and 2 .

\section{The results analysis}

Following the tests at the engine bench as a result of the research methodology presented in Chapter 2, the optimized values for VVT intake and exhaust presented in the tables below were obtained (Tables 1 and 2).

Based on these determinations, the influences on pollutant emissions and dynamic performance for the investigated functional regimes were established. The following influences have been established.

Table 1. Work map for the intake VVT

\begin{tabular}{|c|c|c|c|c|c|c|c|c|c|c|c|c|}
\hline \multirow[b]{2}{*}{ [rpm/mbar] } & \multirow{2}{*}{\multicolumn{3}{|c|}{100014001700}} & \multicolumn{5}{|c|}{$\alpha$ VVT intake $\left[{ }^{\circ} \mathrm{CRK}\right]$} & \\
\hline & & & & 2100 & 2500 & 2800 & 3500 & 4000 & 4800 & 5300 & 6250 & 6500 \\
\hline 330 & 0 & 0 & 0 & $\mathbf{0}$ & $\mathbf{0}$ & 0 & 0 & 5 & 5 & 5 & 0 & 0 \\
\hline 440 & $\mathbf{0}$ & 4 & 2 & 0 & C & $\mathbf{0}$ & 4 & 5 & 6 & 0 & $\mathbf{0}$ & $\mathbf{0}$ \\
\hline 550 & $\mathbf{0}$ & 10 & 10 & 10 & 6 & 5 & 9 & 15 & 9 & 8 & $\mathbf{0}$ & $\mathbf{0}$ \\
\hline 660 & 5 & 20 & 20 & 20 & 10 & 8 & 13 & 15 & 9 & 8 & $\mathbf{0}$ & $\mathbf{0}$ \\
\hline 770 & 10 & 25 & 25 & 25 & 21 & 20 & 20 & 20 & 9 & 8 & $\mathbf{0}$ & $\mathbf{0}$ \\
\hline 880 & 10 & 30 & 29 & 28 & 25 & 25 & 28 & 22 & 12 & 11 & $\mathbf{0}$ & $\mathbf{0}$ \\
\hline 1000 & 15 & 24 & 27 & 27 & 29 & 30 & 28 & 22 & 12 & 11 & 0 & 0 \\
\hline
\end{tabular}

Table 2. Work map for the exhaust VVT

$\alpha$ VVT exhaust $\left[{ }^{\circ} \mathrm{CRK}\right]$

\begin{tabular}{|c|ccccccccccccc|}
{$[\mathrm{rpm} / \mathrm{mbar}]$} & $\mathbf{1 0 0 0}$ & $\mathbf{1 4 0 0}$ & $\mathbf{1 7 0 0}$ & $\mathbf{2 1 0 0}$ & $\mathbf{2 5 0 0}$ & $\mathbf{2 8 0 0}$ & $\mathbf{3 5 0 0}$ & $\mathbf{4 0 0 0}$ & $\mathbf{4 8 0 0}$ & $\mathbf{5 3 0 0}$ & $\mathbf{6 2 5 0}$ & $\mathbf{6 5 0 0}$ \\
\hline $\mathbf{3 3 0}$ & $\mathbf{2 5}$ & $\mathbf{2 5}$ & $\mathbf{2 5}$ & $\mathbf{2 5}$ & $\mathbf{2 5}$ & $\mathbf{2 5}$ & $\mathbf{2 5}$ & $\mathbf{2 7}$ & $\mathbf{2 9}$ & $\mathbf{2 5}$ & $\mathbf{5}$ & $\mathbf{0}$ \\
$\mathbf{4 4 0}$ & $\mathbf{3 0}$ & $\mathbf{3 2}$ & $\mathbf{3 8}$ & $\mathbf{4 0}$ & $\mathbf{4 0}$ & $\mathbf{4 1}$ & $\mathbf{4 0}$ & $\mathbf{3 5}$ & $\mathbf{3 5}$ & $\mathbf{3 5}$ & $\mathbf{1 0}$ & $\mathbf{0}$ \\
$\mathbf{5 5 0}$ & 35 & 35 & $\mathbf{3 8}$ & $\mathbf{4 1}$ & $\mathbf{4 1}$ & $\mathbf{4 1}$ & $\mathbf{4 1}$ & $\mathbf{4 0}$ & $\mathbf{3 9}$ & 37 & $\mathbf{1 0}$ & $\mathbf{0}$ \\
$\mathbf{6 6 0}$ & 25 & 35 & $\mathbf{3 8}$ & $\mathbf{4 0}$ & $\mathbf{4 1}$ & $\mathbf{4 1}$ & $\mathbf{3 6}$ & $\mathbf{3 0}$ & $\mathbf{3 0}$ & $\mathbf{2 9}$ & $\mathbf{1 0}$ & $\mathbf{0}$ \\
$\mathbf{7 7 0}$ & $\mathbf{2 5}$ & $\mathbf{3 3}$ & $\mathbf{3 8}$ & $\mathbf{4 0}$ & $\mathbf{4 0}$ & $\mathbf{3 8}$ & $\mathbf{2 7}$ & $\mathbf{2 0}$ & $\mathbf{2 0}$ & $\mathbf{1 9}$ & $\mathbf{5}$ & $\mathbf{0}$ \\
$\mathbf{8 8 0}$ & $\mathbf{2 5}$ & $\mathbf{2 5}$ & $\mathbf{2 2}$ & $\mathbf{2 0}$ & $\mathbf{2 0}$ & $\mathbf{2 0}$ & $\mathbf{2 0}$ & $\mathbf{1 6}$ & $\mathbf{1 0}$ & $\mathbf{8}$ & $\mathbf{3}$ & $\mathbf{3}$ \\
$\mathbf{1 0 0 0}$ & $\mathbf{2 6}$ & $\mathbf{2 9}$ & $\mathbf{1 7}$ & $\mathbf{1 6}$ & $\mathbf{1 5}$ & $\mathbf{1 3}$ & $\mathbf{1 1}$ & $\mathbf{8}$ & $\mathbf{5}$ & $\mathbf{4}$ & $\mathbf{3}$ & $\mathbf{2}$ \\
\hline
\end{tabular}

\subsection{The influence on the dynamic performance}

To highlight the influence of the double VVT on the effective power and engine torque. The full load regime is followed, in the speed range, with zero VVT position and optimized VVT on the engine bench, these results are represented by the external characteristic shown graphically in figure 5 . 


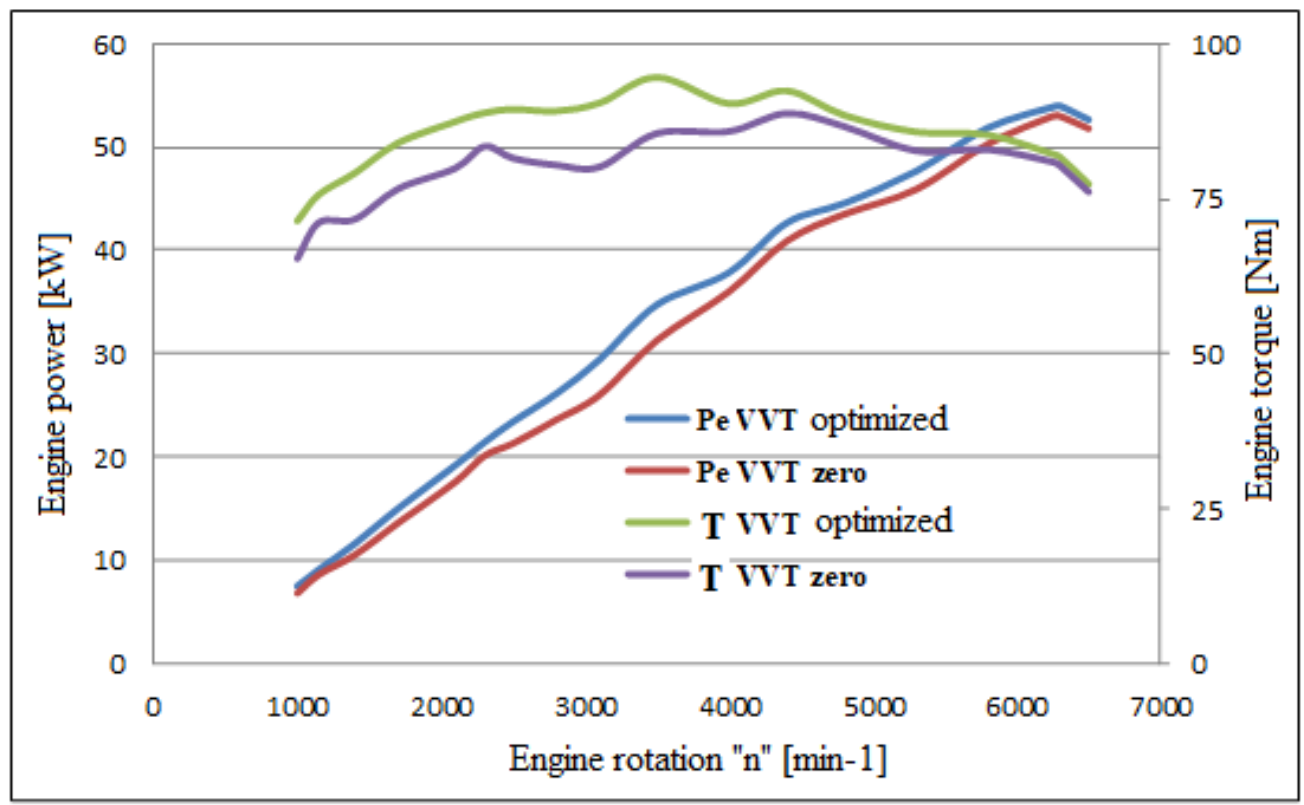

Figure 5. External characteristic for zero VVT and optimized VVT

The following are noted:

- In the speed range $1000 \ldots \mathbf{3 5 0 0} \mathbf{m i n}^{-1}$, which also includes the maximum torque of the engine in the bench $\left(\boldsymbol{n}_{\text {Mmax }}=\mathbf{3 5 0 0} \mathbf{m i n}^{-\mathbf{1}}\right)$,there is a significant increase in engine torque from an average $77,5 N \times m$ value in the case of zero VVT to a value $85 \boldsymbol{N} \times \mathbf{m}$ after we have optimized the VVT positions. An overall increase in engine torque is obtained, with $\mathbf{8}, \mathbf{8} \%$;

- in the speed range $4000 \ldots \mathbf{6 5 0 0} \mathbf{m i n}^{-1}$, which also includes the maximum power speed

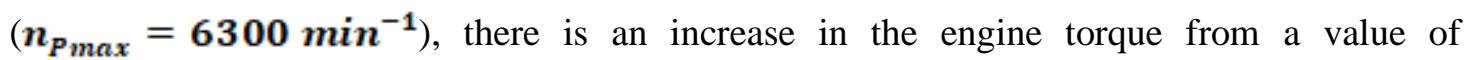
$83 \boldsymbol{N} \cdot \boldsymbol{m}$ in the case of zero VVT to the value of $85,5 \mathbf{N} \cdot \mathbf{m}$, an approximate increase of $3 \%$, a significantly lower gain than the previous range. Therefore, experimental research has confirmed that improved filling has resulted in high performance at high speeds, with VVT positions tending to zero;

- if we refer to the entire external characteristic of the studied engine, there is an overall average increase of $\mathbf{6 , 2 5} \%$ of the engine torque, calculated as the arithmetic mean of the values obtained for the 12 speed regimes investigated;

- for the maximum torque speed, $\boldsymbol{n}_{\text {Mmax }}=\mathbf{3 5 0 0} \mathbf{m i n}^{-\mathbf{1}}$, by optimizing the VVT positions, an increase of $\mathbf{1 0 , 8} \%$ was obtained;

- also at full load of the engine was raised the graph of variation of the filling efficiency (figure 6). For this parameter, a medium gain of $4.7 \%$ is observed in the optimized version of the VVT positions. 


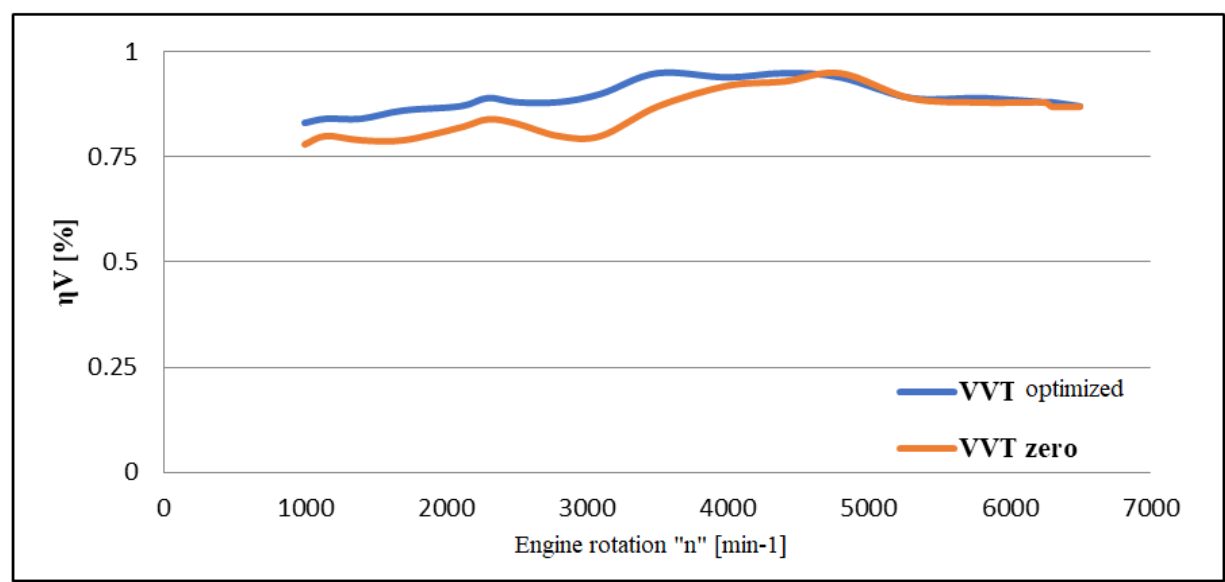

Figure 6. The influence of VVT optimization on filling efficiency at full load

\subsection{The influence on NOx concentrations}

Following experimental determinations, significant influences of combined VVT on nitrogen oxide emissions were highlighted, one of the exhaust gases particularly difficult to reduce in the context of depollution of internal combustion engines. The following were observed:

- on the speed range $1000 \ldots 3100 \mathbf{m i n}^{-1}$ and on the entire load range, the reduction of NOx emissions for optimized VVT is on average overall of $\mathbf{2 3} \%$, so a gain of about a quarter for the variant with optimized VVT;

- on the range $\mathbf{3 5 0 0 . . . 5 8 0 0 ~} \mathbf{m i n}^{-\mathbf{1}}$, the range of regimes with an important weight on the WLTC test cycle, also in the area of partial loads, was obtained an average decrease of only $\mathbf{1}, \mathbf{2} \%$ specifying that this speed zone is called in the research / development activity transit area from emissions at dynamic performance, so here the interest is higher to achieve higher performance;

- In the $6250 \ldots \mathbf{6 5 0 0} \mathbf{m i n}^{-1}$ gap, in the case of the optimized VVT there is a slight increase in nitrogen oxide emissions with $\mathbf{4 0 4} \mathbf{~ p p m}$, area of regimes with extremely low weight in the operation of an SIE, the primary goal being to achieve increased maximum power performance;

- in order to highlight the special qualities of NOx reduction by applying the combined VVT, the relative reduction "RR" (relative gain) calculated with the relation was determined: $(($ NOx VVT optimized - NOx VVT zero) / NOx VVT zero) $) * 100$. The graph in Figure 7 further confirms the advantages offered by the combined VVT system.

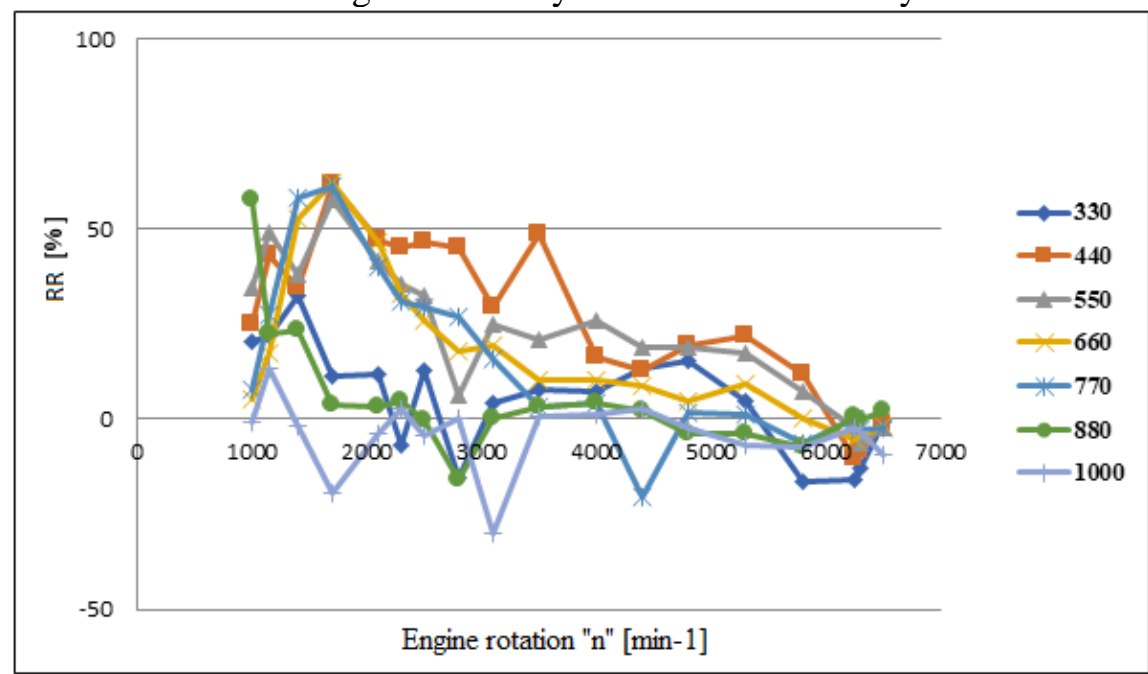

Figure 7. Relative reductions in NOx concentrations between optimized VVT and zero VVT 


\section{Conclusions}

Experimental research has shown that the combined VVT system (intake \& exhaust) can lead to notable advantages both in terms of increasing dynamic performance and in terms of reducing NOx concentration.

After the emissions-performance transition area, where only the increase in power is of interest, NOx emissions suffered a slight increase, insignificant in relation to the overall gain obtained over the entire range of speeds and loads considered.

Also, in the speed range most often encountered on the WLTC test cycle, important gains were obtained in terms of engine torque and filling coefficient.

At the same time, it was highlighted that in the area of maximum power regimes, the position of the valves in order to obtain the peak dynamic performances must be as close as possible to zero.

Finally, we are entitled to state that the combined VVT system is even more stringent in the case of S.I.E. supercharged. Because the sweeping phenomenon appears in them, which requires a much more severe control.

\section{References}

[1] Heisler, H., Advanced Engine Technology, SAE, Hardbound, 2005

[2] Plint, J., Martyr, T., Engine testing , Theory \& Practice, SAE, Casebound,2007

[3] Khair, M., Majewski, A., Diesel emissions and their control, SAE, Hardbound,2006

[4] Ivan, F., Lita D., BUSOI A. Methods and means of depollution of automobile engines, Edit.

MATRIX Publishing House, Bucharest,2013.

[5] *** ENSPM, Course notes, 2009 